International Journal of Engineering \& Technology, 7 (4.24) (2018) 82-86
International Journal of Engineering \& Technology
SPC
Website: www.sciencepubco.com/index.php/IJET
Research paper

\title{
Upfc Compensated Transmission Line Fault Location Based on Travelling Wave Theory and Wavelet Modulus Maxima
}

\author{
K. Durga Syam Prasad ${ }^{*}$, K. Sravanthi ${ }^{2}$, Dr. Ch. Sai Babu ${ }^{3}$ \\ ${ }^{1}$ Department of EEE, Vignan's Institute of Engineering for Women, JNT University, Kakinada. \\ ${ }^{2}$ Assistant Professor, Department of EEE, Vignan's Institute of Information Technology, JNT University, Kakinada. \\ ${ }^{3}$ Professor, Department of EEE, JNT University, Kakinada, India
}

\begin{abstract}
Location of fault in a transmission line which includes flexible AC transmission system (FACTS) devices is a difficult task. This paper comprises of unified power flow controller (UPFC). The implementation control technique for UPFC devices set up dynamic problems in power system that must be reflecting on issues related to power system protection. The occurrence of a fault in the fault loop containing a UPFC affects transient and steady-state parameters of voltage and current in the power system. In this paper the identification of fault section before or after UPFC for a transmission line with wavelet fuzzy logic system discriminator. Wavelet technique extracts features, and the faulty location is found when the normalized features are fed to the fuzzy logic system. As soon as the fault section is located, the control shifts to that and is analyzed basing on the representation of the traveling wave theory through wavelet modulus maxima. This criterion detects the faulty section with respect to FACTS device very accuratelyin terms of distance from relay point. The above-said method is simulated for various fault situations and locations with wide changes in the conditions of operation in the presence of UPFC.
\end{abstract}

Keywords: Unified Power Flow Controller, Wavelet Modulus Maxima, fuzzy logic systems and Fault Location.

\section{Introduction}

In thepresentenvironment, transmission line systems are designed for extensive power transfer capability. This can increase focus on constraints of theTransmission line by means of asudden change in constraint can be reduced.FACTS Device can provide flexible reinforcement approach.[1] One of the most important and most versatile devices is unified power flow controller. In this device, it consists of two voltage sources converters, linked to the main circuit by means of shunt and series transformers which provides an exclusive combination of rapid compensation by series \&shunt devices.The UPFC provide new scope for the system parameter control, like bus voltage, active and reactive power, by the using this device increases the stability of the system andimprovestransferring capability of power and other problem occurred during protection of power system.

Some exploration has been done to decide the fault area utilizing Kalman channel strategy [2] for FACTS remunerated line utilizes varieties in current signs amid transient conditions with respect to issues. This gives the fault area as far as line reactance and protection. Then again, the Kalman separating approach discovers its restriction in that fault protection can't be displayed and it requires an alternate number of channels to accomplish the undertaking. Then again, this technique has its downside that fault protection can't be demonstrated and it requires an adjustment in the quantity of channels to accomplish the assignment. Finding the fault separation rapidly and being dependable is as yet a major test. Heading out waves identified with shortcomings change with time and recurrence.[3] Unadulterated recurrence space techniques sometimes fall short for those transient signs. Distinguishing the area and the kind of fault happened on the power framework is a troublesome errand to be proficient both precisely and all the while. This paper presents fault area utilizing Wavelet Technique, by utilizing voyaging wave hypothesis and its switch voltage wave ideas.[4] It additionally demonstrates wavelet method for remove figuring of different sorts of issues in the transmission framework. The execution of wavelet modulus maxima system is seen by ascertaining separation.[5]

\section{Wavelet Transform Decomposition}

A lot of data in regards to issues is joined with the signs of homeless people. So these signs are utilized to find the shortcomings of segments or transmission lines. [6] It additionally manages the sort of fault and area, arrangement of shortcomings and breaks down its motivation. In this way it enhances the dependability of the framework. Transient signs have high-recurrence attributes. Contrasted with other scientific flag investigation procedures, the Wavelet changes more equipped for transient signs. [7] As a rule, multi-upheaval decay strategy is utilized for wavelet change under different scales. It channels the flag with a low-pass channel and high-pass match. The approximations coefficients are the segments of the low recurrence of the flag created by sifting it by a low-pass channel and the high-scale,. The points of interest are high-recurrence, parts of the low recurrence of the flag delivered by separating the flag by a high-pass channel. Those two channels have break even with data transmission. After each decay, the testing recurrence is diminished significantly. At that point recursively disintegrate the low pass channel yields (approximations) to get the following stage segment $[8,9]$. 
A discrete signal $\mathrm{x}(\mathrm{n})$ is given which is being fast transformed at instant $\mathrm{k}$ and scale $\mathrm{j}$,

$\mathrm{Dj}(\mathrm{k})$ coefficient has a high-frequency component

$\mathrm{Aj}(\mathrm{k})$ coefficient has a low-frequency component

$D_{j}(K):\left[2^{-(j+1)} f_{s}, 2^{-j} f_{s}\right]$

$A_{j}(K):\left[0,2^{-(j+1)} f_{s}\right]$

Where $f_{s}$ represents sampling frequency and $j=1,2,3 \ldots \mathrm{m}$ The main sequence of signal $\mathrm{X}(\mathrm{n})$ is the sum of all $\mathrm{Dj}(\mathrm{k})$ and $\operatorname{Aj}(\mathrm{k})$.

$X(n)=D_{1}(n)+A_{1}(n)$

$=D_{1}(n)+D_{2}(n)+A_{2} m$

$=D_{1}(n)+D_{2}(n)+D_{3}(n)+A_{3}(n)$

After decomposition, coefficient wavelet modulus Maxima (WMM) is defined as the absolute local maximum values of wavelet coefficients. The WMM is noticeably at a point of the TimeAmplitude response; the amplitude indicates thechange in edge intensity whichindicates thefrequencyof the contentsignal.

\section{Test System}

A typical transmission system is shown in the Figure. The test system is a two area system. In this two three-phase sources are used,and five loads are used on the system model. One fault blocks are used on this model to observe the fault location with wavelet modulus maxima technique and differential equation based algorithm methods.

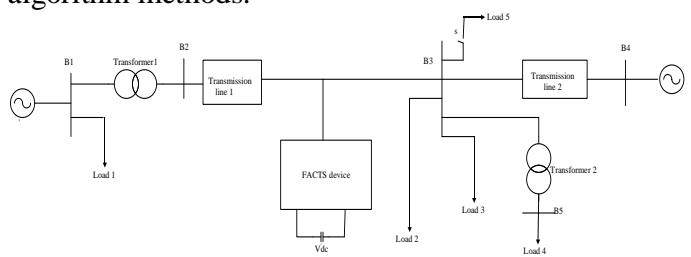

Fig. 1. UPFC compensated transmission line model

The test system parameters are at area1 source rated voltage of $13.8 \mathrm{kV}$ and its short circuit capacity 21,000 MVA similarly at area 2 source having rated voltage of $735 \mathrm{kV}$ and its SC capacity 30,000 MVA.These two areas are connected with atransmission line having resistance 0.001 p.u. and reactance: 0.0195 p.u. Sending end transformer $(\mathrm{D} / \mathrm{Y})$ connected rated voltage $13.8 / 735 \mathrm{kV}$, rated power $2100 \mathrm{MVA}$, leakage resistance 0.002 p.u. and leakage reactance 0.08 Similarly receiving end transformer $(\mathrm{Y} / \mathrm{Y})$ rated voltage $735 / 230 \mathrm{kV}$, rated power 300 MVA, leakage resistance 0.002 p.u. leakage reactance 0.15 p.u.The test system is consisting of five loads at different places $100 \mathrm{MW}$ load 1 is connected at bus 1 , three loads are connectedat bus 3, those loads 2 and 3 are 1.32 MW, 330MVAR and load 5 having 300MW. Load 4 is connected near to bus 5 having capacity $250 \mathrm{MW}$. UPFC device ishaving twocontrollers linked to the main circuit through theshunt and series transformers which offer an exclusive combination of fast compensation by SSSC and STATCOM devices each one rated power 100 MVA, Nominal DC voltage $20 \mathrm{kV}$ and Nominal AC voltage $13.8 \mathrm{kV}$.

\section{Feature Extraction}

Fault section identification before and after UPFC can be done by using feature extraction of thewavelet transform; the current wave occurred for fault (post-fault current with half cycle) is processed by WT and Y1,and Y2 are found as features of coefficients of wavelets. As this transform is the best tool for analysis of fault transient and extraction of the feature is done by pre-processing the signal of current through WT.

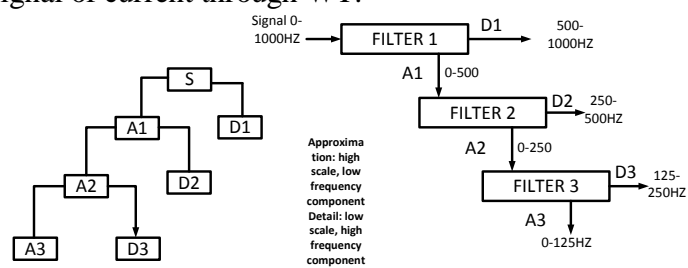

Fig.2. Wavelet Decomposition Concept

The Daubechies wavelets 4 is selected for thetransient signal for this analysis. The given signal is decomposed into three levels. This consequences in level 1 approximation A1 $(0-500 \mathrm{~Hz})$ and detail D1 $(500-1000 \mathrm{~Hz}), \quad$ similarly in level 2 approximation A2 $(0-250 \mathrm{~Hz})$ and detail D2 $(250-500 \mathrm{~Hz})$, and in level 3 approximation A3 $(0-125 \mathrm{~Hz})$ and detail D3 $(125-$ $250 \mathrm{~Hz}$ )

The value of Y1 is calculated theratio of detailed coefficients energy (D2) to the approximate coefficients energy (A3). The value of Y2 is calculated theratio of detailed coefficients energy (D1) to the approximate coefficients energy (A3). Hence, Y1 and Y2 are evidence for the component harmonic concerning fundamental components.

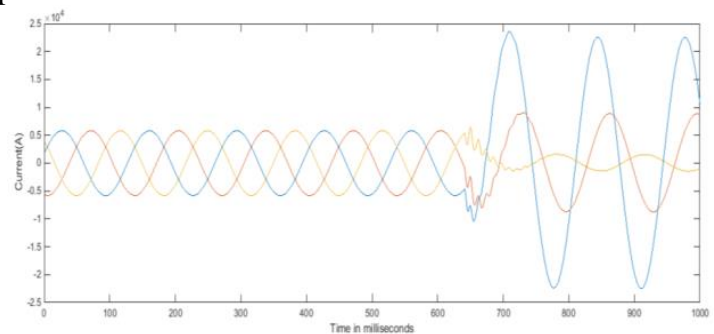

Fig. 3. Three phase current waveforms during LG fault before the UPFC. The above waveform shows the line-to-ground fault on a transmission line. Moreover, it happened before the FACTs device.

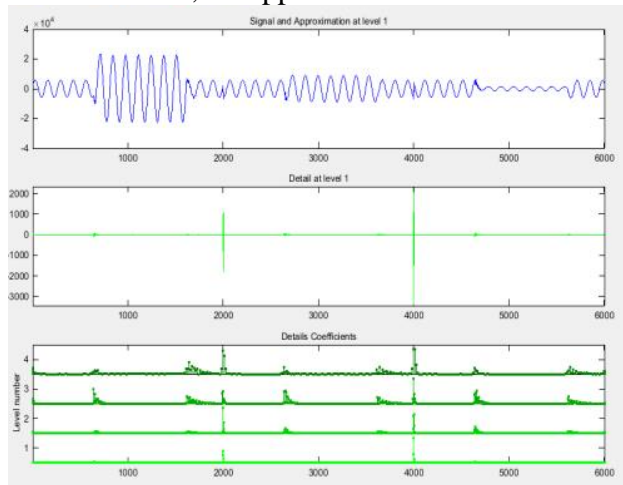

Fig. 4. Approximate and detailed coefficients of three-phase currentwaveforms during LG fault before theUPFC.

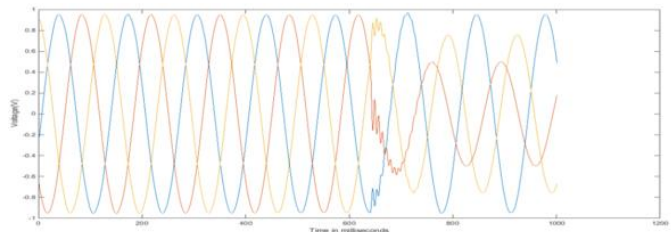

Fig. 5. Three phase current waveforms during LL fault before the UPFC.

The transmission line with UPFC, have high harmonic components like the $3^{\text {rd }}, 5^{\text {th }}$ and $7^{\text {th }}$ are highly pronounced and hence for fault section identification, thisabove feature extraction of wavelet transform are extracted for ultimate categorization by using thefuzzy logic system. The particular values of Y1 and Y2 are normalizedand given to the fuzzy logic system to obtain the fault section identification. From table 1 shows the values of $\mathrm{Y} 1$ 
and $\mathrm{Y} 2$ for different types of afault such as $\mathrm{AG}, \mathrm{AB}, \mathrm{ABG}, \mathrm{ABC}$ and $\mathrm{ABCG}$ faults taking place before and after the UPFC.
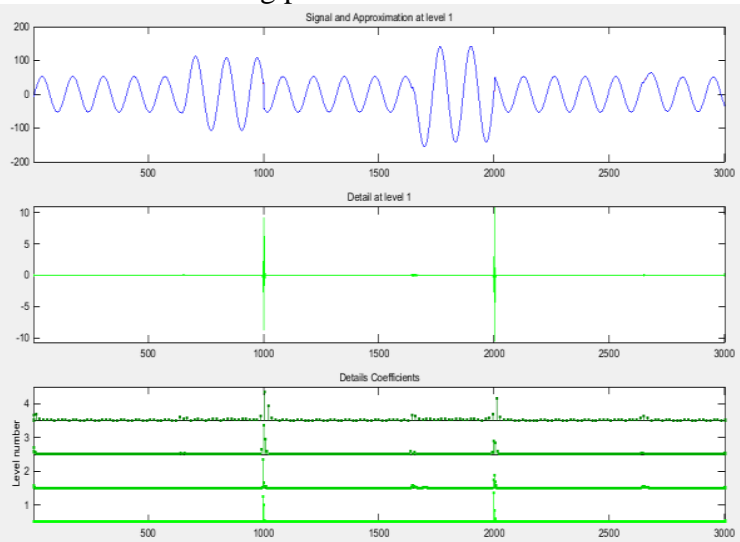

Fig. 6. Approximate and detailed coefficients of three-phase curren waveforms during LL fault before theUPFC

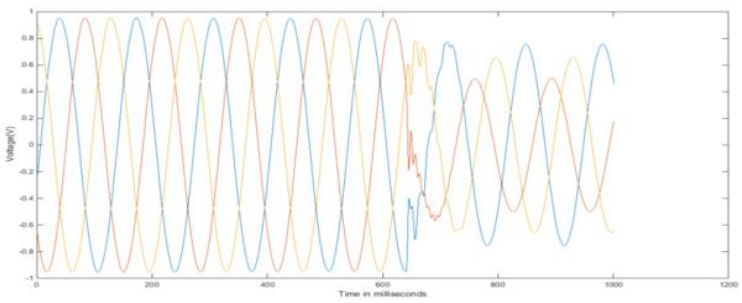

Fig. 7. Three phase current waveforms during LLG fault before the UPFC.

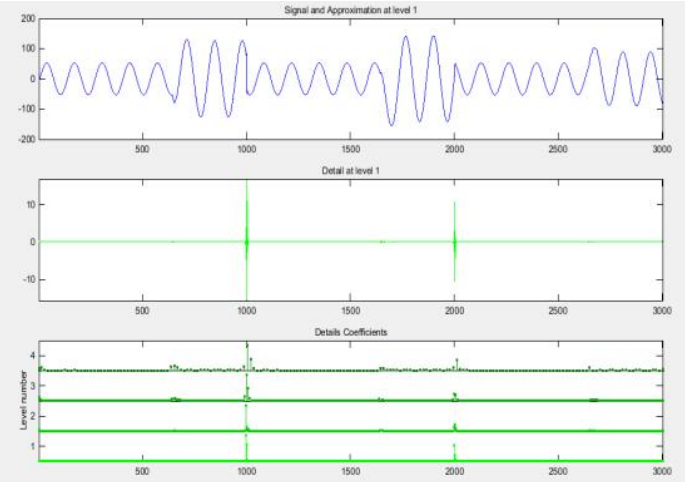

Fig. 8. Approximate and detailed coefficients of three-phasewaveforms of current during LLG fault after the UPFC

Table 1. Y1 and Y2 values before after UPFC

\begin{tabular}{|l|l|l|l|l|l|}
\hline \multicolumn{2}{|l|}{ Fault location } & \multicolumn{2}{l|}{} & \multirow{2}{*}{ Y1 } & \multirow{2}{*}{ Y2 } \\
\cline { 1 - 4 } Length in \% & Fault & Rf $(\boldsymbol{\Omega})$ & Angle $^{\mathbf{o}}$ & & \\
\hline 10 & AG & 5 & 20 & 0.3067 & 0.4286 \\
\hline 30 & AG & 5 & 20 & 0.3252 & 0.4489 \\
\hline 45 & AG & 5 & 20 & 0.2972 & 0.4168 \\
\hline 70 & AG & 5 & 20 & $\mathbf{0 . 7 9 3 1}$ & $\mathbf{0 . 8 1 1 3}$ \\
\hline 10 & AB & 10 & 30 & 0.3652 & 0.4258 \\
\hline 30 & AB & 10 & 30 & 0.2565 & 0.3547 \\
\hline 45 & AB & 10 & 30 & 0.2256 & 0.2687 \\
\hline 70 & AB & 10 & 30 & $\mathbf{0 . 6 5 8 8}$ & $\mathbf{0 . 7 4 1 2}$ \\
\hline 10 & ABG & 15 & 40 & 0.3422 & 0.3856 \\
\hline 30 & ABG & 15 & 40 & 0.2456 & 0.2113 \\
\hline 45 & ABG & 15 & 40 & 0.1986 & 0.2078 \\
\hline 70 & ABG & 15 & 40 & $\mathbf{0 . 7 1 2 2}$ & $\mathbf{0 . 8 2 5 7}$ \\
\hline 10 & ABC & 10 & 20 & 0.3564 & 0.4569 \\
\hline 30 & ABC & 10 & 20 & 0.3478 & 0.4325 \\
\hline 45 & ABC & 10 & 20 & 0.2985 & 0.3547 \\
\hline 70 & ABC & 10 & 20 & $\mathbf{0 . 7 8 1 2}$ & $\mathbf{0 . 8 0 1 2}$ \\
\hline 10 & ABCG & 15 & 30 & 0.3685 & 0.5644 \\
\hline 30 & ABCG & 15 & 30 & 0.3547 & 0.4578 \\
\hline 45 & ABCG & 15 & 30 & 0.2588 & 0.3458 \\
\hline 70 & ABCG & 15 & 30 & $\mathbf{0 . 7 4 5 8}$ & $\mathbf{0 . 8 2 1 4}$ \\
\hline
\end{tabular}

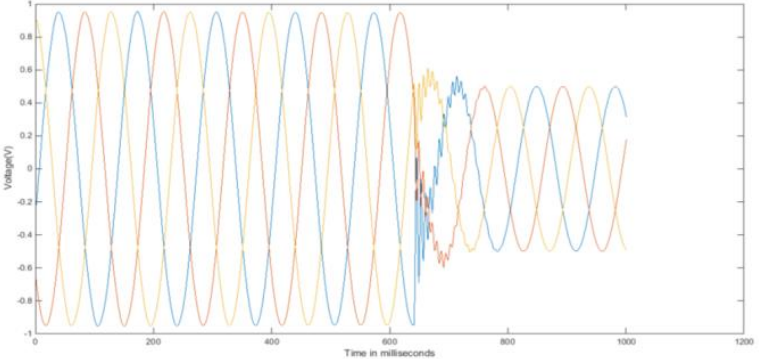

Fig. 9. Three phase current waveforms during LLL fault after the UPFC.

Different fault conditions at $10 \%, 30 \%, 50 \%$ \& $70 \%$ length of the transmission line distances, $20^{\circ}, 30^{\circ} \& 40^{\circ}$ of load angles and fault resistances at $5 \Omega, 10 \Omega$ and $15 \Omega$ are tested. For an AG fault at $50 \%$ of thedistance of theline, the values of $\mathrm{Y} 1$ and $\mathrm{Y} 2$ are 0.2972 and 0.4168 , correspondingly. For fault occurring at $70 \%$ of the considered line, the respective values are 0.7931 and 0.8113 . Similar observations are made for an $\mathrm{AB}, \mathrm{ABG}, \mathrm{ABC}$ and $\mathrm{ABCG}$ fault which occurs prior and after the UPFC. The values of Y1 and Y2 are higher for faults after the UPFC compared to those for faults before the UPFC. After completion of extraction of feature by using a wavelet transform, the automatic fault section identifier is designed using a fuzzy logic system.

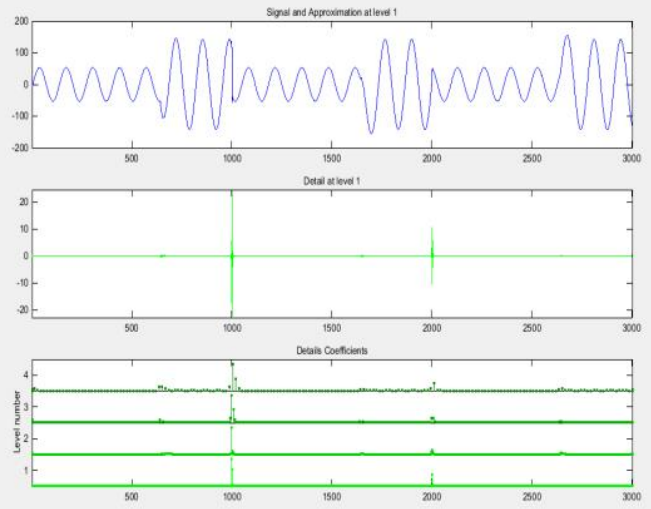

Fig. 10. Approximate and detailed coefficients of three-phase waveforms of current during LLL fault after the UPFC.

The fuzzy logic system is designed using the information of $\mathrm{AG}, \mathrm{AB}, \mathrm{ABG}, \mathrm{ABC}$ and $\mathrm{ABCG}$ conditions of fault. Next, a fuzzy IF-THEN rule base is made for fault section identification. The fuzzy logic system has been designed as follows. For an ' $M$ ' class categorization with ' $n$ ' feature vectors, the rule is $L j$, If $Y 1$ is $P_{j 1}$ and $\mathrm{Yn}$ is $\mathrm{P}_{\mathrm{jn}}$, then the class is $\mathrm{Mj}$, where $\mathrm{j}=1,2,3 \mathrm{~N}$ and $\mathrm{Y}=(\mathrm{Y} 1$, $\mathrm{Y} 2,, \mathrm{Yn})$. Here, $\mathrm{P}_{\mathrm{ji}}$ is the linguistic value, ' $\mathrm{M}$ ' is the class and ' $\mathrm{N}$ ' is the fuzzy IF-THEN rules. The gradation of the rule $\mathrm{Lj}$ is determined by the following operation

$\mu \mathrm{j}(\mathrm{x})=\min \{\mu \mathrm{j} 1(\mathrm{x} 1), \mu \mathrm{j} 2(\mathrm{x} 2), \quad, \mu \mathrm{jn}(\mathrm{xn})\}$

where $\mu \mathrm{ji}(\mathrm{xi})$ represents membership function of the linguistic value Pji. Without a certainty grade [13], the respective pattern can be classified by the single winner rule Ljp, defined as

$\mu \mathrm{j}(\mathrm{x})=\max \{\mu \mathrm{j}(\mathrm{x}), \mathrm{j}=1,2,3, \ldots . \mathrm{N}\}$

The features $\mathrm{Y} 1$ and $\mathrm{Y} 2$ are fuzzified with a triangular membership function. The triangular membership function forY1 is shown in Fig. 3, where the input is done into three levels, low (L), medium $(\mathrm{M})$ and high $(\mathrm{H})$. Similar membership is defined for Y2. The fuzzified inputs are given to a fuzzy rule base having nine rules. 


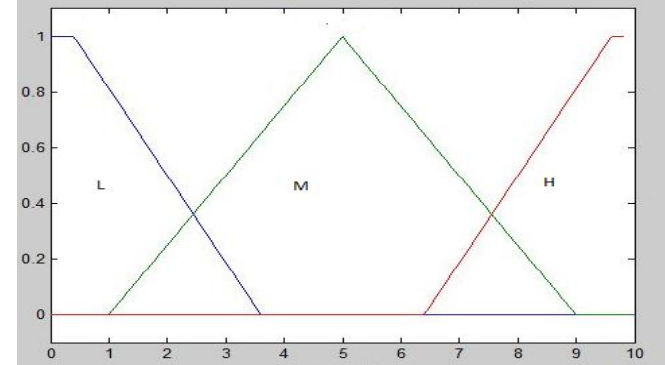

Fig .11. Triangular membership function

Table 2. Fuzzy rules

\begin{tabular}{|l|l|l|l|}
\multicolumn{4}{|c}{ Table 2. Fuzzy rules } \\
\hline \multirow{2}{*}{ S.no } & Classes & \multirow{2}{*}{ Rule } \\
\cline { 2 - 3 } & Y1 & Y2 & \\
\hline 1 & L & L & Fault occurs before the UPFC \\
\hline 2 & L & M & Fault occurs before the UPFC \\
\hline 3 & L & H & Fault occurs before the UPFC \\
\hline 4 & M & L & Fault occurs before the UPFC \\
\hline 5 & M & M & Fault occurs after the UPFC \\
\hline 6 & M & H & Fault occurs after the UPFC \\
\hline 7 & H & L & Fault occurs before the UPFC \\
\hline 8 & H & M & Fault occurs after the UPFC \\
\hline 9 & H & H & Fault occurs after the UPFC \\
\hline
\end{tabular}

Table 3. Fuzzy logic system output for DFT and wavelet

\begin{tabular}{|l|l|l|l|l|l|}
\hline Fault location & \multicolumn{5}{|l|}{ fuzzy logic system } \\
\hline Length in $\%$ & Fault & $\operatorname{Rf}(\Omega)$ & Angle $\delta^{\circ}$ & DFT & wavelet \\
\hline 10 & AG & 5 & 20 & 0 & 0 \\
\hline 30 & AG & 5 & 20 & 0 & 0 \\
\hline 45 & AG & 5 & 20 & 0 & 0 \\
\hline 70 & AG & 5 & 20 & $\mathbf{1}$ & $\mathbf{1}$ \\
\hline 10 & AB & 10 & 30 & 0 & 0 \\
\hline 30 & AB & 10 & 30 & 0 & 0 \\
\hline 45 & AB & 10 & 30 & 0 & 0 \\
\hline 70 & AB & 10 & 30 & $\mathbf{1}$ & $\mathbf{1}$ \\
\hline 10 & ABG & 15 & 40 & 0 & 0 \\
\hline 30 & ABG & 15 & 40 & 0 & 0 \\
\hline 45 & ABG & 15 & 40 & 0 & 0 \\
\hline 70 & ABG & 15 & 40 & $\mathbf{0}$ & $\mathbf{1}$ \\
\hline 10 & ABC & 10 & 20 & 0 & 0 \\
\hline 30 & ABC & 10 & 20 & 0 & 0 \\
\hline 45 & ABC & 10 & 20 & 0 & 0 \\
\hline 70 & ABC & 10 & 20 & $\mathbf{0}$ & $\mathbf{1}$ \\
\hline 10 & ABCG & 15 & 30 & 0 & 0 \\
\hline 30 & ABCG & 15 & 30 & 0 & 0 \\
\hline 45 & ABCG & 15 & 30 & 0 & 0 \\
\hline 70 & ABCG & 15 & 30 & 0 & 1 \\
\hline
\end{tabular}

The obtained results from table 2, when input data is given to thefuzzy logic expert system, for each input set, one of the nine rules are fired. The resultant output is one for fault after compensation device and zeroes for afault before fault compensation device. For $\mathrm{AG}$ fault resistance $5 \mathrm{ohms}$ and delta $=20$ degrees at $10 \%$, $30 \%$ and $45 \%$ of the line. The fuzzy logic system provides zero output and indicates that the fault appeared prior the UPFC. Similarly, the fault resistance $5 \mathrm{ohms}$ and delta $=20$ degrees at 705 of the line, the fuzzy logic system provides one as the output. This indicates the fault appeared after UPFC. The projected fuzzy logic system has been tested for $\mathrm{AB}, \mathrm{ABG}, \mathrm{ABC}$, and $\mathrm{ABCG}$ at $10 \%$, $30 \%, 45 \%$ and $70 \%$ of the total line length with the load angles of 10 degrees, 30 degrees, and 40 degrees. The test system is tested for extreme cases, and it provides accurate results. The above system is also tested for discrete Fourier transform system and fuzzy logic system for fault location identification. The results obtained by DFT are compared with theproposed wavelet transform, and thefuzzy logic system is shown in table 2. In comparison, it is found that DFT cannot locatefaulty section for FACTS compensated transmission line.

\subsection{Fault Distance Based On Wmma}

The test system is tested for AG fault at a distance of $100 \mathrm{~km}$ from the relay point. The test system simulating for AG line fault is shown in Fig. 2.3. The fault voltage waves and current waves of an AG line fault are shown in Fig. $4.6 \&$ 4.7. The reverse voltage traveling wave for the $\mathrm{AC}$ fault line is done by equation 4.1.

The output voltage and the reverse voltage traveling wave for the faulty line is done by using

$\mathrm{Vr}=[\mathrm{Vac}-\mathrm{Zc} * \mathrm{iac}] / 2$

Where,

$\mathrm{Vr}=$ voltage reverse travelling wave.

Vac $=$ voltage obtained during normal operation Iac $=$ current obtained during normal operation

$\mathrm{Zc}=$ surge impedance of the transmission line.

$\mathrm{RV}$ traveling wave appears in the line only when,

$\mathrm{Zc}>\mathrm{Zt}$ or $\mathrm{Zc}<\mathrm{Zt}$

$\mathrm{Zc}$ denotes characteristic impedance of the transmission line.

$\mathrm{Zt}$ is the terminating impedance of the transmission line.

So by using the wavelet toolbox we analyzed the reverse voltage traveling wave and by taking details and approximations, we analyzed $\mathrm{d} 1$, and by using the respective formulae, we calculated the fault distance.

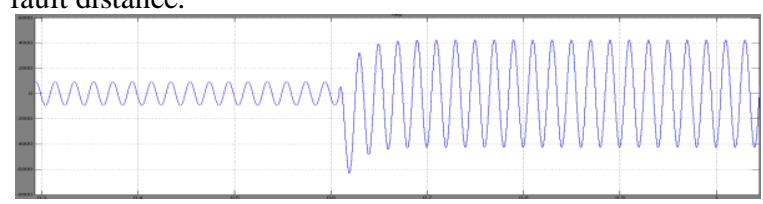

Fig.12. Current signal during single LG fault

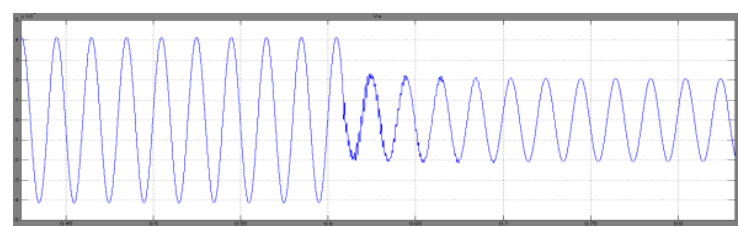

Fig.13. Voltage signal during single line to ground fault

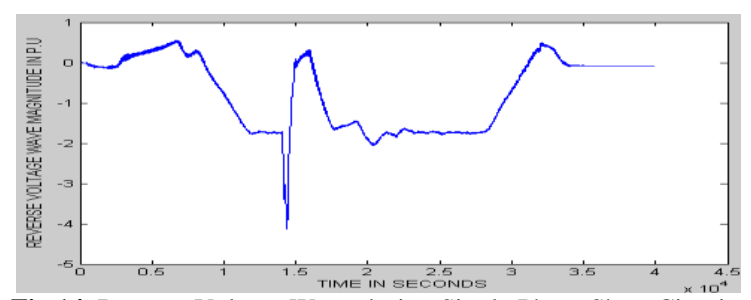

Fig.14. Reverse Voltage Wave during Single Phase Short Circuit

The fault takes place at $100 \mathrm{~km}$ from the three-phase source from 0.6 to 0.65 seconds for 0.05 seconds. In the simulation diagram, the block parameters and the breaker's switching times are kept between 0.6 to 0.65 seconds. The wavelet modulus maxima which are obtained for faulted signal in a single LG fault are shown.

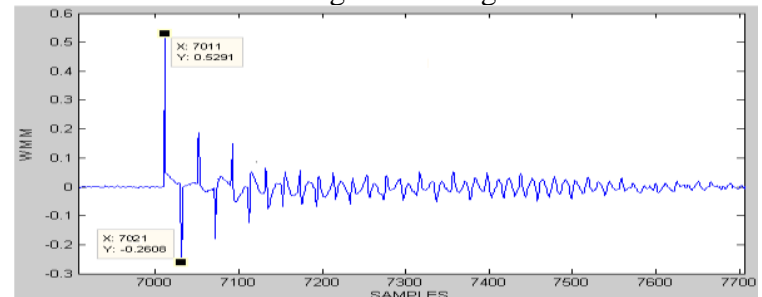

Fig. 15. Wavelet modulus maxima signal for line to ground fault

\begin{tabular}{|l|l|l|l|l|l|l|}
\hline \multirow{2}{*}{$\begin{array}{l}\text { Faul } \\
\text { t } \\
\text { type }\end{array}$} & \begin{tabular}{l} 
FFT \\
\cline { 2 - 6 } \\
estimat- \\
length(k
\end{tabular} & $\begin{array}{l}\text { \% er- } \\
\text { ror }\end{array}$ & $\begin{array}{l}\text { Estimat- } \\
\text { ed } \\
\text { length(k }\end{array}$ & $\begin{array}{l}\text { \% } \\
\text { er- } \\
\text { ror }\end{array}$ & $\begin{array}{l}\text { Estimat- } \\
\text { ed } \\
\text { length(k }\end{array}$ & $\begin{array}{l}\text { \% er- } \\
\text { ror }\end{array}$ \\
\hline
\end{tabular}




\begin{tabular}{|l|l|l|l|l|l|l|}
\hline & m) & & m) & & m) & \\
\hline LG & 289.7 & -3.4 & 298.1 & -1.9 & 299.75 & -0.079 \\
\hline LL & 297.7 & -2.3 & 298.3 & -1.7 & 299.82 & -0.173 \\
\hline LLL & 302.1 & 2.1 & 301.4 & 1.4 & 300.134 & 0.1034 \\
\hline LLG & & & & & 299.33 & -0.67 \\
\hline
\end{tabular}

From the table 4, it is clear that wavelet transform gives to detect, locate the location a fault. WT technique can assist to the conventional wave analyzers used in the power transmission lines to denoise the transmitted signals. This method is a better one to detect the presence of faults in the networks.

\section{Conclusion}

In this paper, the location of fault for transmission lines FACTs device, aFuzzy logic system with Wavelet transformwere introduced. In the above one can observe that the fault occurred before the facts device or after the facts device is verified with DFT technique,but wallet transforms identified very accurately where it happened whether after or before the FACTs device.Moreover, the wavelet modulus maxima calculate the exact fault distance form the source side.

\section{References}

[1] Zhang S, Zou G, Huang Q, 2018, A Traveling-Wave-Based Fault Location Scheme for MMC-Based Multi-Terminal DC Grids, mdpi.com.

[2] Ye S, Wellner R, 2017, Rapid and Consistent Identification of Stratigraphic Boundaries and Stacking Patterns in Well-logs-An Automated Process Utilizing Wavelet Transforms and Beta, onepetro.org.

[3] Yang X, Gao F, 2017, Exact traveling wave solutions for the local fractional two-dimensional Burgers-type equations, Elsevier.

[4] Tang L, 2018, A traveling wave differential protection scheme for the half-wavelength transmission line, Elsevier.

[5] Tang L, Dong X, Luo S, 2017, A new differential protection of transmission line based on the equivalent traveling wave, ieeexplore.ieee.org.

[6] Stanislaus R, 2017, Electron transit-time and exit velocity in linear beam traveling wave amplifier for a dielectric rod supported tapehelix slow wave structure, e-fermat.org.

[7] Smaoui G, Young A, Abid M, 2017, Single Scale CWT Algorithm for ECG Beat Detection for a Portable Monitoring System, Journal of Medical and Biological Engineering, vol. 37, no. 1, pp. 132-139.

[8] Smaoui G, Young A, 2017, Single Scale CWT Algorithm for ECG Beat Detection for a Portable Monitoring System, Springer.

[9] Shiji T, Remya S, 2017, Computer Aided Segmentation of Breas Ultrasound Images Using Scale Invariant Feature Transform (SIFT) and Bag Of Features, Elsevier.

[10] Seadawy A R, 2017, Travelling-wave solutions of a weakly nonlinear two-dimensional higher-order Kadomtsev-Petviashvili dynamical equation for dispersive shallow-water waves, The European Physical Journal Plus, vol. 132, no. 1, p. 29-31.

[11] Martin A M, Burridge C P, Ingram J, Fraser T A, Carver S, 2018 , Invasive pathogen drives host population collapse: Effects of a travelling wave of sarcoptic mange on bare-nosed wombats, Journal of Applied Ecology, vol. 55, no. 1, pp. 331-341.

[12] Ma Y, Fang B, Ding Q, Wang F, 2018, Analysis of Mold Friction in a Continuous Casting Using Wavelet Transform, Metallurgical and Materials Transactions B, 221.

[13] DurgaSyam Prasad K, DrSaibabu C, 2016, Detection, Classification, Protection and Mitigation of Power System Faults and Disturbances Using Wavelets Transform, vol. 9, no. 29, pp. 313-327. 